\title{
On evolving chains of cube-free binary sequences
}

\author{
Jacek M. Kowalski ${ }^{1}$ and Andrzej Pȩkalski ${ }^{2}$ \\ ${ }^{1}$ Department of Physics, University of North Texas \\ Denton TX 76293, United States \\ e-mail: jacek.kowalski@unt.edu \\ ${ }^{2}$ Institute of Theoretical Physics, University of Wrocław \\ 50-203 Wrocław, Poland \\ e-mail: ja.pekalscy@gmail.com
}

\begin{abstract}
Chains of concatenated finite binary words are considered, where each word, except possibly the very first one, is composed of alternating blocks of zeroes and ones with block lengths not exceeding two. These chains are formed following two evolution schemes. The first scheme is standard, where alternating blocks are visited at random. In the second approach, proposed by us in this paper, each subsequent word of the chain is uniquely determined by its immediate predecessor, being formed as a specifically inflated version of that word. Famous Kolakoski sequence is then just one, very special example of such deterministic evolution when one starts from its third element. We present heuristic arguments supported by simulations indicating that all such deterministic infinite chains should have the asymptotic density of digit 1 equal $1 / 2$ and that the subsequent word lengths asymptotically scale with factor of $3 / 2$ and hence the density of 1 's in subsequent finite words may also tend to $1 / 2$.
\end{abstract}

Keywords: Automatic sequences, Random and deterministic evolution of binary words, Algorithmic combinatorics, Kolakoski and related sequences, Scaling law.

2020 Mathematics Subject Classification: 11B83, 11B85, 11R45, 11Y55, 65B10, 68R15.

\section{Introduction}

Almost all observations presented in this paper will be formulated as properties of binary sequences with elements $\sigma \in\{0,1\}$, though in some cases we will turn to the alphabets $\{1,2\}$ or $\{-1,1\}$. 
In particular, for the $\{1,2\}$ alphabet one uses $\tau=1+\sigma$ transformation rule and for the $\{-1,1\}$ alphabet of Ising spin variables one uses $\nu=2 \sigma-1$ transformation rule. The basic notation and definitions mostly follow those used in the monographs $[1,4,13] . w_{k}$ will always be a finite binary word labelled with an integer $k,\left|w_{k}\right|$ is the number of digits in it (word's length) and $\| w_{k}||$ is the number of digits 1 within that word. If $w_{k}$ consists of digits 0 and 1 , then $\left\|w_{k}\right\|$ is its $l_{1}$ norm. Binary word can be concatenated into finite chains $w_{1} w_{2} w_{3} \ldots w_{M}$ of words of total length $\sum\left|w_{k}\right|$. This sum and related sums below run from $k=1$ till $k=M$. The density of a particular word $w_{k}$ is defined as

$$
d\left(w_{k}\right)=\frac{\| w_{k}||}{\left|w_{k}\right|}
$$

and that of a finite chain as

$$
d\left(w_{1} w_{2} \ldots w_{M}\right)=\frac{|| w_{1} w_{2} \ldots w_{M}||}{\sum\left|w_{k}\right|}=\frac{\sum d\left(w_{k}\right)\left|w_{k}\right|}{\sum\left|w_{k}\right|} .
$$

Of particular interest for infinite chains generated in some procedures is the existence of the limit of $d\left(w_{1} w_{2} w_{3} \ldots w_{M}\right)$ for $M \rightarrow \infty$. In terms of the binary variable used below for subsequent digits of built words, it is equivalent to the problem of the Cèsaro summability of corresponding infinite series $\sum_{k=1}^{\infty} \sigma_{k}$.

For the corresponding one-sided Ising chains one is concerned with the existence of the average magnetisation per spin of the system.

Two simple observations are important in this context:

a) If an infinite chain, for which this limit exists, is modified into another infinite chain by augmenting it at the beginning with an arbitrary (finite) word $w_{0}$ or by dropping any finite number of its initial words, then both modifications will have the same limiting behaviour as the original chain.

b) The existence of the limit of partial densities $d\left(w_{k}\right)$ for $k \rightarrow \infty$ implies the existence of the limit of $d\left(w_{1} w_{2} w_{3} \ldots w_{M}\right)$ for $M \rightarrow \infty$. It is also well known that the converse statement is not true - particular densities may endlessly fluctuate around some value with overall infinite chain density still convergent to some limit.

We will concentrate on a widely studied class of binary sequences not containing sub-sequences 000 and 111, that is free of cubes of single digit words 0 and 1 . These sequences may still contain cubes $w w w$ of some other concatenated binary words $w$ of length $\geq 2$. Binary sequences which do not contain cubes of any finite binary word $w$ are also called cube-free in the literature, but they of course constitute much more restricted family of binary sequences within which, for example, periodic infinite sequences of any period are excluded by definition.

We will begin our analysis of evolutions of binary words of our class with their equivalent interpretation as walks on graphs from Section 2, followed then by natural in this context, standard analysis of completely random evolutions. Stationary distributions giving information about expected digit densities in such infinite walks are also of interest.

Complementary to randomly created chains are infinite chains of words created according to some deterministic evolution rules defining the chain, and where each word in the chain contains 
a complete prescription for the creation of its immediate successor. The following Section 3 discusses such a model, proposed by us, where subsequent words are both cube-free and typically have increasing length, being specifically inflated versions of their predecessors. We conjecture that the described there specific inflation procedure results in limiting densities equal $1 / 2$ for any starting initial binary word and we support the claim by some numerical evidence and related estimates. Two of such evolutions happen to lead to infinite chains of words coinciding with the famous Kolakoski-Oldenburger sequence [16,19], if one modifies one or two their initial elements. The relationship of our deterministic inflation scheme with alternating morphisms prescription, originally specifically proposed for the Kolakoski sequence in [9], is discussed in the Appendix. Mentions and comments on some other well-known sequences generated by morphisms are again delegated to the Appendix. In Section 4 we present numerical results for densities of long chains, illustrate the observed asymptotic scaling law for word lengths in such chains and following from it convergence of particular word densities. The concluding Section 6 contains some incentives for further studies of the problem and a proposal for a possible graphical illustrations of the inflation process on 2-D square lattices.

\section{Cube-free words as walks on a graph. Random evolutions}

Sequences from our family of sequences could be put into one to one correspondence with walks on a four-vertex graph with vertices marked by just four basic words $0,11,00$ and 1 as shown on Figure 1.

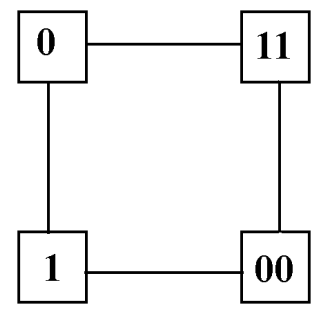

Figure 1. Basic graph for cube-free sequences

These basic words will also be called blocks or runs in the rest of the text. An obvious observation is that in all walks on this graph, blocks composed of zeroes and ones must alternate, i.e., each block of zeroes could only be followed by a block of ones, and vice versa.

Let $N \geq 1$ be the length of a binary word formed by a concatenation of $k \geq 1$ vertex words in the order of visiting. Clearly $N$ could be larger than $k$ if some two-letter words are included in a given walk. Denoting by $k_{0}, k_{1}, k_{00} k_{11}$ the numbers of particular types of vertices visited in a walk of $k-1$ steps, one has:

$$
N=k_{0}+k_{1}+2\left(k_{00}+k_{11}\right) \text {. }
$$

Conversely, any finite binary word of length $N$ not containing cubes 000 and 111 can be uniquely represented by a walk on a graph by partitioning it from its beginning into subsequent sub-words (our blocks) consisting of one or two identical symbols. The number of vertices visited in such a walk is then clearly equal $N-\left(k_{00}+k_{11}\right)$. 
Let $F_{N}$ be the total number of such words of length $N$. One may notice that $F_{N}$ are elements of the Fibonacci sequence with generating elements $F_{1}=2, F_{2}=4$, satisfying the standard recurrence relation:

$$
F_{N+2}=F_{N}+F_{N+1} \text {. }
$$

Indeed, among all sequences of length $N+2$ one may consider first those ending with words 00 or 11 which could be obtained by concatenating any word of length $N$ ending with 0 with 11 and all ending with 1 with 00 . The other classes of words of length $N+2$ ending, respectively, with words 01 or 10 can be obtained by adding to words from the set of words of the length $N+1$ a single complementary digit ( 1 to follow terminal digit 0 , and 0 to follow terminal digit 1 ). This observation for cube-free sequences has been apparently known for a long time, but for a more general, recent discussion of related problems see also [3].

Some additional combinatorial results, which can be readily obtained from the graph representation, are related to adjacency matrices of the graph from Figure 1. For any cyclic labelling of graph vertices (like $0,11,00,1 ; 11,00,1,0 \ldots$ or $0,1,00,11 ; 1,00,11,0, \ldots$ ) all these matrices look the same being $4 \times 4$ circulant matrices which can be written in the block form:

$$
\Omega_{x}=\left(\begin{array}{ll}
\sigma_{x} & \sigma_{x} \\
\sigma_{x} & \sigma_{x}
\end{array}\right) \quad \text { where } \quad \sigma_{x}=\left(\begin{array}{ll}
0 & 1 \\
1 & 0
\end{array}\right)
$$

is one of the Pauli matrices. The standard result of the graph theory amounts here to the statement that the numbers of $M$-steps distinct walks joining two given vertices of the Figure 2 are given by the respective matrix elements of the $\Omega_{x}^{M}$.

We are now ready for the analysis of random walks on the graph from Figure 2 announced in the Introduction. Let us identify vertices of the graph from Figure 1 with four states and let the transition probability along the edge joining states $(0)$ and (1) be denoted by $p$. In the related Markov chain the transition probabilities along edges $\{(1),(00)\}$ and $\{(0),(11)\}$ must both be equal $1-p$ and hence the transition probability along the edge $\{(00),(11)\}$ is also equal $p$. The transition matrix $T$ of such Markov chain has the form of a circulant:

$$
\left(\begin{array}{cc}
(1-p) \sigma_{x} & p \sigma_{x} \\
p \sigma_{x} & (1-p) \sigma_{x}
\end{array}\right) .
$$

Since our graph (Figure 2) is a regular one with $\mathrm{m}=4$ vertices (i.e., having equal degrees of all vertices, in our case equal two) the stationary distribution formula for random walks on such graphs (see, e.g., [23]):

$$
p_{s}=\frac{\text { vertex degree }}{2 m}
$$

determines components of the stationary distribution all equal $1 / 4$. This also can be verified for simple particular transition matrix of Eq. (6) by straightforward calculation. In conclusion, it means that for any transition probability $p$ the limiting density of 0 and 1 symbols is equal $1 / 2$, as intuitively expected.

Our cube-free sequences of length $N=3 k, k=1,2, \ldots$ can also be partitioned into subsequent words of length three. There are six $((2+4)$, as indicated by Eq. (4)) such words: 001, 010, 001 and $101,110,011$, which can be considered as representing a subset of vertices of a 3-D unit 
cube. Concatenation of these words into our cube-free words leads to restrictions imposed on vertex-to-vertex transitions. All allowed transitions can be represented by a digraph with six vertices connected by six oriented, six bi-directional edges and having six vertex loops. A standard but lengthier analysis of the related Markov processes with equally probable out-routes from each vertex (vertex loops counted with multiplicity one) again results in the limiting stationary density of 1 's equal $1 / 2$.

Two other classes of walks can be obtained after eliminating one of length two blocks from the graph on Figure 1. One may still consider the remaining parts as linear graphs: $0-1-00$ or $1-0-11$. Random walks on them with equal $1 / 2$ transition probabilities from the central vertex to its neighbours lead to stationary density of 1 equal, respectively, $1 / 3$ and $2 / 3$ as expected. However deterministic cube-free chains formed from words $0,1,11$ or $0,1,00$ may still have interesting irrational infinite chain densities (like Fibonacci word, see Appendix and the modified inflation rules there).

\section{Deterministic evolution of cube-free words}

We start by looking at the ordered pair of the first and last letter of the word $w_{1}$ (these may coincide if one starts with a word of length 1). This pair is used to determine the first block of being created word $w_{2}$ : pair $(0,0)$ produces 1 , pair $(0,1)$ produces 0 , pair $(1,0)$ produces block 11 and pair $(1,1)$ produces block 00 . In the next step, one takes an ordered pair of the second (if any) letter of the word $w_{1}$ and the last created letter of $w_{2}$ and uses the same rule as above to determine the second block of $w_{2}$. This second block will always be of opposite (alternate) type with respect to already created first block of $w_{2}$ regardless of the actual type $x$ of the second letter of $w_{1}$. Indeed, $(x, 0)$ results in 1 or 11 and $(x, 1)$ produces 0 or 00 . This procedure is being repeated for all, if any, subsequent letters of $w_{1}$. After completion of the $w_{2}$ build-up, this word is considered as a second stage evolution program for the subsequent word $w_{3}$, etc. The entire procedure can also be summarized as follows: zeroes from the preceding word never produce 00 blocks in the next word created but become conditionally transformed into a single digit 0 or 1 . On the other hand, ones from the preceding word always transform into blocks 00 or 11 depending on the type of lastly created letter of the next word.

Examples (with semicolons added in concatenations for reader's convenience):

1. $w_{1}=011, w_{2}=01100, w_{3}=1001101, \ldots$ concatenated into: $w_{1} w_{2} w_{3} \ldots=011 ; 01100 ; 1001101 ; \ldots$,

2. $w_{1}=100, w_{2}=1101, w_{3}=1100100, \ldots$ concatenated into: $w_{1} w_{2} w_{3} \ldots=100 ; 1101 ; 1100100 ; \ldots$,

3. $w_{1}=0101, w_{2}=011011, w_{3}=0110010011, \ldots$ concatenated into: $w_{1} w_{2} w_{3} \ldots=0101 ; 011011 ; 0110010011 ; \ldots$,

4. the case when $w_{1}$ is not cube-free: $w_{1}=1000, w_{2}=11010, w_{3}=11001001$ concatenated into: $w_{1} w_{2} w_{3} \ldots=1000 ; 11010 ; 11001001 ; \ldots$,

5. another not cube-free $w_{1}=0000, w_{2}=1010, w_{3}=110110$ concatenated into: $w_{1} w_{2} w_{3} \ldots=0000 ; 1010 ; 110110 ; 1100100110 \ldots$ 
It is important to notice that in this evolution words $w_{n+1}$ are specifically inflated versions of the preceding word $w_{n}$ and, conversely, the sequence of digits formed from the reduced by 1 lengths of subsequent blocks of $w_{n+1}$ (in short, reduced runs or block lengths) always coincide with the preceding sequence $w_{n}$ in the chain. For illustration of this rather obvious feature, please read backwards all given above examples. Additionally, words with the norm $\left\|w_{k}\right\| \geq 1$ always evolve here into words of longer length:

$$
\left|w_{k+1}\right|=\left|w_{k}\right|+|| w_{k}||
$$

This particular feature of the above described word inflation scheme leads to a compact expression for the density of an entire finite chain of $M$ words. Indeed, re-writing Eq. (8) as $\left|w_{k+1}\right|-\left|w_{k}\right|=$ $\left\|w_{k}\right\|$ and summing them over $k$ from 1 to $M$, gives:

$$
\sum_{k=1}^{M}|| w_{k}||=\left|w_{k+1}\right|-\left|w_{k}\right| .
$$

Returning to the definition of density of the chain $w_{1} w_{2} \ldots w_{M}$, Eq. (2), one obtains

$$
d\left(w_{1} w_{2} \ldots w_{N}\right)=\frac{\left|w_{N+1}\right|-\left|w_{1}\right|}{\sum_{k=1}^{M}\left|w_{k}\right|} .
$$

The last formula shows that the only information needed to determine the density of 1 in a concatenated finite chain of words formed using any inflationary evolution satisfying Eq. (8), is stored in the sequence of these words lengths. It also suggests that for the ratios of subsequent words lengths $\left|w_{k+1}\right| /\left|w_{k}\right|$ asymptotically scaling with a factor $\gamma>1$, the finite chain densities (Eq. (10)) of concatenated words must tend to $\gamma-1$ for $M \rightarrow \infty$. Indeed, for $\left|w_{k+1}\right| /\left|w_{k}\right| \rightarrow \gamma$, $d\left(w_{k}\right)=|| w_{k}|| /\left|w_{k}\right|=\left(\left|w_{k+1}\right|-\left|w_{k}\right|\right) /\left|w_{k}\right| \rightarrow \gamma-1$.

We expect that scaling behaviour with a particularly simple, rational factor of $3 / 2$, is indeed present in all infinite chains formed using above-described inflation rule when applied to an arbitrary initial word.

\subsection{Remark on backward compression}

As indicated before, any second word $w_{2}$ in a chain created in our evolution scheme from an arbitrary finite word $w_{1}$ is a cube-free word of the length $N=\left|w_{2}\right|=\left|w_{1}\right|+|| w_{1}||$ being, in general, one of the $F_{N}$ such words. Initial word $w_{1}$ can then be restored from $w_{2}$ by writing reduced block length sequence of $w_{2}$. If, however, $w$ is an arbitrary cube-free word of length $N$, an attempt of finding its possible predecessor in the described evolution process by writing the sequence $w^{\prime}$ of its reduced block lengths, may lead to a predecessor which itself is not a cube-free, or such that the concatenated word $w^{\prime} w$ cannot be obtained in the described inflation procedure from the word $w^{\prime}$. Examples: $w=010101, w^{\prime}=000000$ not being cube-free; $w=11011$, $w^{\prime}=101$ and $w$ cannot be restored by inflation of $w^{\prime}$. The reader may also experiment with all

$10=4+6$ cube-free words of length 4 and conclude that half of them may not be re-created from their compressed version rule, and two can only be preceded by words which are not cube-free. 
This observation allows elimination of many words from the entire community of $F_{N}$ cube-free words of length $N$ all those words which cannot be created from particular shorter cube-free words in the described inflation process. This is also a route to procedures allowing obtaining tight estimates of an infinite chain density under assumption of its existence (see references in the Appendix).

\subsection{Complement words and mirrored evolutions}

Any finite or infinite binary word $w$ can be transformed into its complement $w^{c}$ by re-coding: $1 \Leftrightarrow 0$. Clearly

$$
\|w\|+|| w^{c}||=|w|+\left|w^{c}\right| .
$$

One can also interchange the role of symbols 0 and 1 in our evolution rule and carry out such mirrored evolution starting from any finite initial word $w_{1}$. Clearly, words created from an arbitrary finite word $w$ in our evolution scheme are complement words of words obtained in mirrored evolution from $w^{c}$.

Moreover, if an infinite word obtained in an evolution from an arbitrary finite cube-free word has some limiting density $p$ of entries 1 , then its complement is also cube-free and must have the density of entries 1 equal $1-p$.

All of the above can also be illustrated by a following model. Let us assume that one has an unlimited supply of domino tiles $\quad \mathrm{O}^{\circ}$ and starts with arranging some finite number of them into an array with all tiles in vertical position (physicists may think of arrays made of spin vectors) and continues the build-up of a chain with our inflation rules guided by symbols, say, in the bottom row (with a dot on a tile considered as 1 ).

\subsection{Two special evolutions starting from a single digit}

Let us now pay more attention to the two simplest evolutions starting with words 0 or 1 created according to our rules. These are:

$$
\begin{array}{ll}
\text { initial word } 0 \text { evoloving into: } & 0 ; 1 ; 00 ; 10 ; 110 ; 11001 ; 00110100 ; \ldots \\
\text { initial word } 1 \text { evoloving into: } & 1 \mid 00 ; 10 ; 110 ; 11001 ; 00110100 ; \ldots
\end{array}
$$

Note that these two infinite chains of words coincide if one either drops the first word in Eq. (12a) or precede the first word of Eq. (12b) by word 0 still consistent with the compression scheme for Eq. (12b).

\subsection{Kolakoski-Oldenburg sequence}

Let us augment the sequence from Eq. (12b) with an additional initial digit equal 1, obtaining

$$
110010110110010011010010110010010 \ldots
$$

and partition it after that into subsequent blocks of identical digits separated by colon signs

$$
\text { 11:00:1:0:11:0:11:00:1:00:11:0:1:00:1:0:11:00:1:00:1:0 }
$$


Returning, for a while, to the $\{1,2\}$ alphabet, one may write the sequence (13) as

$$
221121221221121122121121221121121, \ldots
$$

which can be compared to the sequence of its block lengths read from (15):

$$
2211212212211211221211, \ldots
$$

It suggests that both, generated in this way, infinite sequences (13) and (15) do coincide, that is, they are invariant under subsequent block lengths transformation. There is one more binary sequence of that type exhibiting this invariance property which can be obtained by augmenting (13) by yet another initial element 0 to obtain:

$$
0110010110110010011010010110010010, \ldots
$$

which, in the variables of the $\{1,2\}$ alphabet reads as:

$$
1221121221221121122121121221121121, \ldots
$$

The interest in the sequence (18) was triggered by a note by Kolakoski [16], and it bears since then his name. It could also be called the Oldenburger-Kolakoski (O.-K.) sequence since, as it was noticed much later, it had been first considered in a broader context by Oldenburger [19]. A good source of information about the O.-K. sequence is the related Wikipedia page [25] and Wolfram's page [24].

For both versions of the O.-K. sequence (as indicated above, differences in some initial finite fragment of the sequence do not matter in the infinite chain density consideration) Keane [15] conjectured that the asymptotic density of digits 1 in this sequence is equal $1 / 2$. Despite of numerous, often quite sophisticated attempts, no one has succeeded in proving it and even a (quite symbolic nowadays) money prize is assigned to the problem (see [22,24,25] and references therein). However, brilliant efforts of several authors $[5,8,17]$ have succeeded in producing quite tight bounds around $1 / 2$ for the presumed density.

A simple observation that distinct infinite chains of cube-free words can also be generated from an arbitrary finite non-empty binary word via our inflationary evolution rule, lead us to a conjecture that all such chains could all have the limiting chain density equal $1 / 2$, including the possibility of an asymptotic scaling law for word lengths and hence the existence of the limit for subsequent word densities.

\section{Numerical results}

We began by checking first that infinite distinct chains created from few selected test initial words may indeed have the expected behaviour. Results are presented in Figure 2 for several initial words, as marked in the legend. For better readability we have restricted the number of inflation steps in Figures 2 and 5 to 30 instead of 45 used in the simulations. 


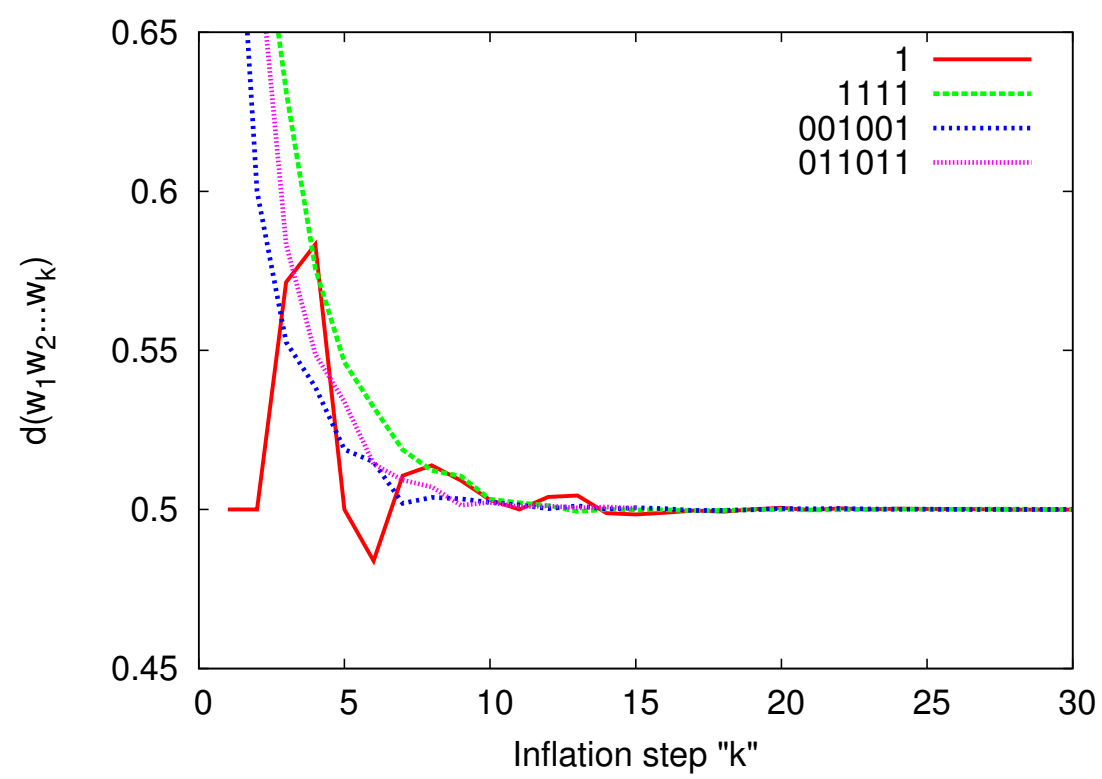

Figure 2. Density of 1's in concatenated words in inflation step $k$

Two of them start from initial cube-free words of length 6 (with the smallest and largest possible initial densities for such cube-free words), two other begin, respectively, with the third element of the classical O.-K. sequence and with an "illegal" initial word 1111, both having word density equal one. The second word in the chain evolved from 1111 has length of 8 and is one of the $F_{8}$ which cannot be present in O.-K. sequences. All calculated chain densities, as expected, exhibit large initial fluctuations but after about 20 evolution steps all approach closely $1 / 2$ with fluctuations smaller than 0.0002 .

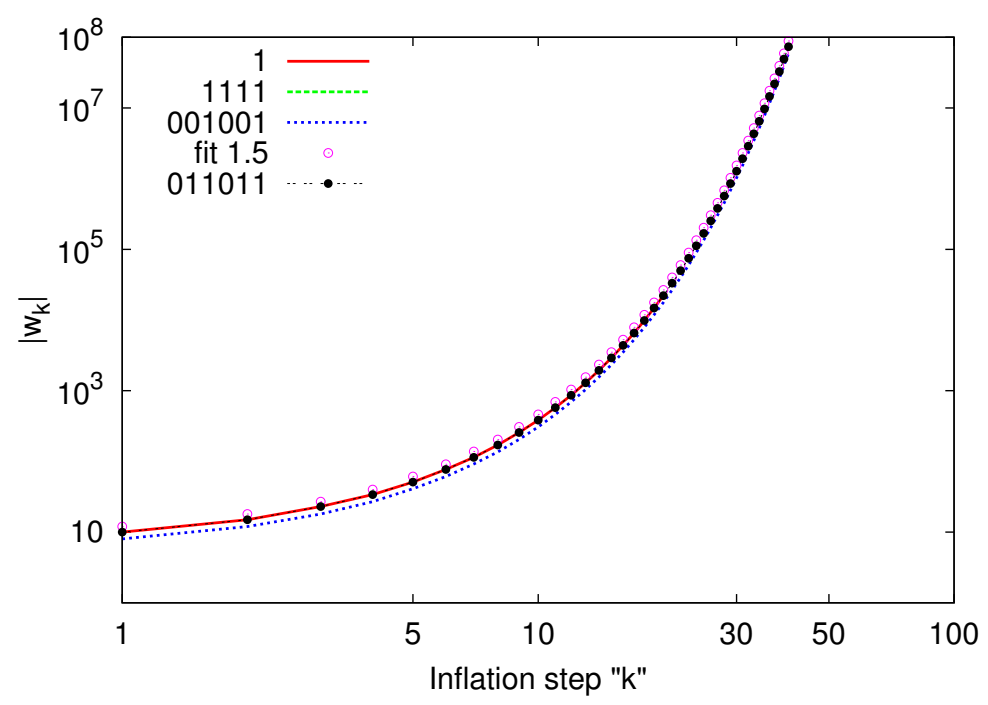

Figure 3. Length of words $\left|w_{k}\right|$ generated in steps $k$

Rate of increase of subsequent word lengths after the $k$-th step of inflation is presented on Figure 3 with used there doubly logarithmic scale and the same choice of initial words as in Figure 2. It is evident that all of them follow quite well expected dependence $\left|w_{k+1}\right| \approx 1.5 \cdot\left|w_{k}\right|$. 
Figure 4 shows the same dependence as in Figure 3 but for concatenated words. Again, the same fit to scaling law as in Figure 3 soon becomes very good in evolutions from longer initial words and, understandably, is more sluggish for the truncated O.-K. sequence, as this is a chain with slowest growth of finite chain lengths.

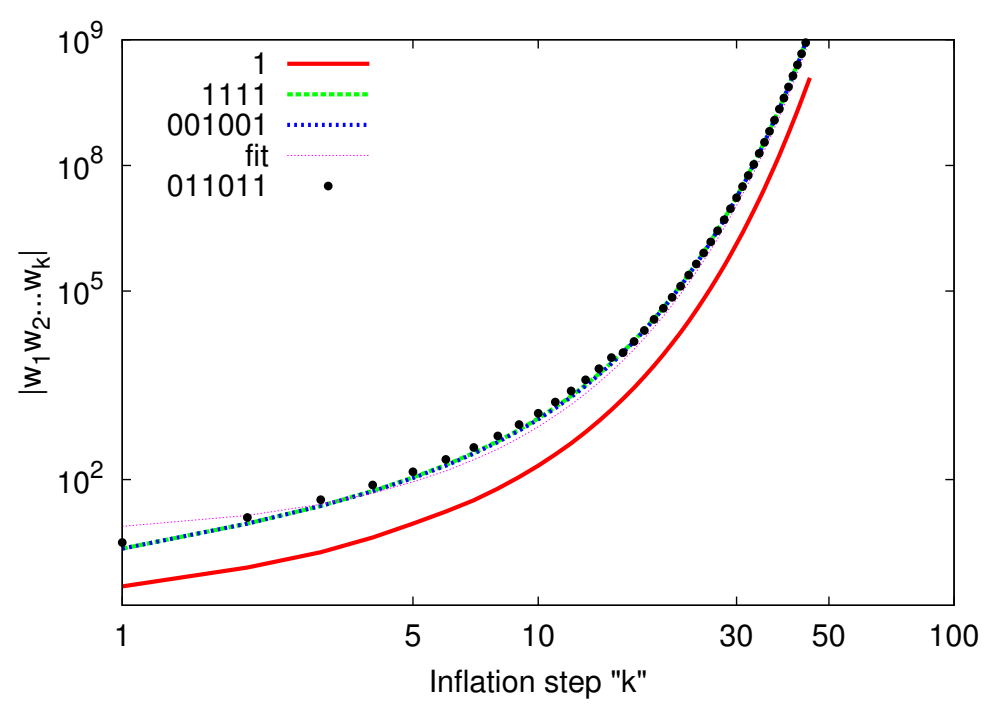

Figure 4. Length of concatenated words $\left|w_{1} w_{2} w_{3} \ldots\right|$ generated in steps $k$

Finally, the behaviour of density of 1's in subsequent inflated, but not concatenated, particular word is shown on Figure 5. As mentioned earlier in Section 3 the presence of asymptotic scaling law with simple, rational factor $3 / 2$ indeed forces their convergence to $1 / 2$.

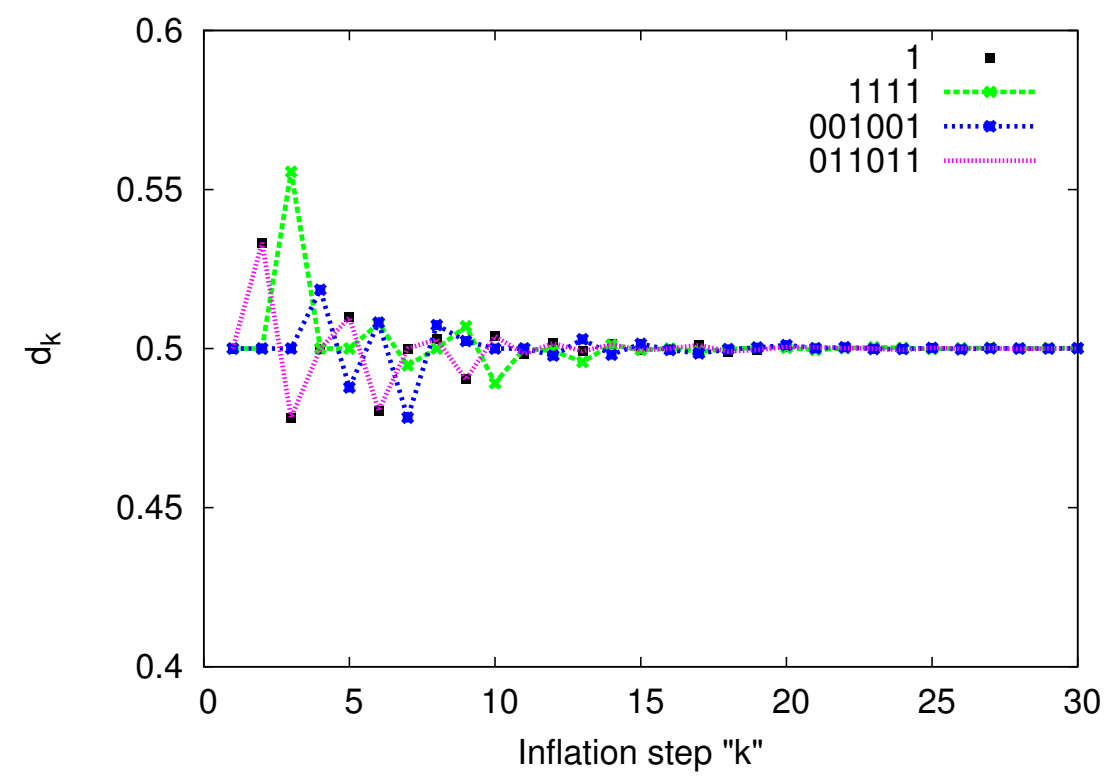

Figure 5. Density of 1's in inflation step $k$ 


\section{Constants characterizing sequences obtained from an arbitrary finite cube-free word in the deterministic expansion scheme}

Any binary word $\sigma_{1} \sigma_{2} \ldots \sigma_{k} \ldots, \sigma_{i} \in\{0,1\}$ can be associated with a binary fraction

$$
0 . \sigma_{1} \sigma_{2} \ldots \sigma_{k} \ldots=\sum_{k} \sigma_{k} \times 2^{-k}, \quad k=1,2, \ldots, \infty .
$$

Binary fraction assigned to the variant of the Kolakowski sequence starting with 11 was calculated as a decimal fraction starting from $0.79450 \ldots$ up to 25000 digits, [22, 24, 25].

One can easily notice that infinite fractions formed from cube-free words are bounded from above by a periodic binary fraction $0.11011011011 \ldots$ (period 110 , representing rational number $6 / 7$, with periodic decimal expansion $0.85714 \ldots$. The corresponding lower bound is given by another periodic binary fraction $0.00100100100 \ldots$ (period 001 , representing rational number $1 / 7$, with periodic decimal expansion $0.14285 \ldots$. Infinite fractions formed in our inflationary scheme starting from any finite cube-free word must then be irrational numbers filling the interval $[1 / 7,6 / 7]$. The original Kolakoski sequence starting from 0 in $\{0,1\}$ alphabet (A000002 in OEIS) is then represented by quoted above expansion, that is by $0.397247 \ldots$.

Just for illustration of the above remarks we calculated short fragments of the binary expansions for words obtained in the inflationary procedure from relatively short initial cube-free words. A sequence starting from 1: 100|10|110|11001|... (A000002 with its first two digits removed), i.e., our sequence (12b) is represented by its decimal fraction $0.58898925712 \ldots$. Four others starting from short cube-free words are: $w_{1}=001001$, which gives $0.1452274 \ldots$, $w_{1}=110110$ for which we got $0.8560665 \ldots, w_{1}=011011$ which gives $0.4280303418 \ldots$ and $w_{1}=100100$, with the fraction equal $0.5753654241 \ldots$. All those values are within the above described limits.

\section{Concluding remarks}

Our original interest in the subject was triggered by its relations to variants of the Ising model [18] and topics as interesting as quantum processors working with one-dimensional arrays of spin states. We demonstrated that deterministic evolution scheme where each word contains intrinsic instruction for the construction of its cube-free, inflated successor may indeed produce a wide class of infinite binary sequences with well-mixed blocks $0,1,00$ and 11 , all of them having the limiting density of 1 equal $1 / 2$. The observed by other authors invariance of just two special, cube-free Kolakoski-type sequences with respect to the "run length" transformation appears then as a special feature, not critical for the limiting density equal $1 / 2$ phenomenon. At the same time alternating morphisms procedure (strictly speaking not of morphism type) originally proposed for the Kolakoski sequence alone, could be still used, after minor modification, as equivalent to our evolution determined by the preceding fragment of chain history. This and some other issues contextually related to our work are discussed in the Appendix. 
We hope that it may revive interest in more detailed studies of limiting densities of sequences which contain infinite chains of cube-free words, in particular those built according to the discussed variant of inflation scheme, for which a kind of the generalized Keane's conjecture can possibly be proved and, at least, density bounds improved. Indeed, these bounds for the O.-K. sequence itself are still of interest, as well as the search for most efficient algorithms (see again [5, 6, 8, $17,22,24,25]$. One should also add that some other, more complex types of self-reading and inflationary sequences were also studied for years by Paun [20], and that some interesting results about cube-free sequences were obtained in $[11,14]$. Graphical representation of such chains of cube-free words on square lattice are worth of interest. We intend to work on self-generating graphical representations of our chains of words provided by tightly wound, decorated spiral self-avoiding walks, originating at some point of the square lattice. One can also blur the difference between deterministic or completely random evolutions by considering them as different ways of decorating the trivial infinite chain $01010101 \ldots$ (of obvious density $1 / 2$ ) with inserts replacing some 1 with blocks 11 and some 0 with blocks 00 . Clearly, alternating insertions schemes of 00 and 11 will trivially leave the density of so created chains unchanged and equal $1 / 2$. However, as one can easily verify, this is not happening in the O.-K. sequence, and apparently not in any of our sequences produced from an arbitrary word, what makes the studies of such possibly asymptotically compensating infinite chains still of some interest.

\section{A Appendix}

\section{A.1 Two limits}

For any finite chain of finite words (not necessarily formed using the evolution rule described in Section 3) and any real constant $a$ one may start from an obvious inequality

$$
\left|d\left(w_{1} w_{2} \ldots w_{M}\right)\right|-a \mid \leq \frac{\sum_{k=1}^{M}\left|d\left(w_{k}\right)-a\right|\left|w_{k}\right|}{\sum_{k=1}^{M}\left|w_{k}\right|} .
$$

It allows to conclude that the convergence of the sequence $\left\{d\left(w_{k}\right)\right\}$ to $a$ implies the convergence of the sequence $\left\{d\left(w_{1} w_{2} \ldots w_{M}\right)\right\}$ to the same limit. For this purpose let us select $L<M$ and separate right hand side of the inequality (A.1) into two sums: one with summation in the numerator up to $L$ and the other one with the summation there starting from $L+1$. The first sum can be estimated from above by

$$
\frac{\sum_{k=1}^{L}\left|w_{k}\right|}{\sum_{k=1}^{M}\left|w_{k}\right|} \text { as } \quad\left|d\left(w_{k}\right)-1\right| \leq 1
$$

for all densities. On the other hand, for any $\epsilon>0$ there exists $L(\epsilon)$ such that the second sum running in the numerator from $L(\epsilon)+1$ can be again estimated from above by, say, $\epsilon / 2$. Simultaneously, for sufficiently large $M>L(\epsilon)$ the first sum could also be made smaller than $\epsilon / 2$, what proves the claim. 


\section{A.2 How deterministic inflation rules may simplify the density questions for some infinite chains}

Less trivial example is provided by the interestingly irregular famous Thue-Morse sequence generated by morphism $\mu(0)=01, \mu(1)=10$ (see, e.g., [1] and an excellent summary of the history of this sequence by the same authors [2]). With the above definition subsequent sequence elements may be generated by iterations $\mu^{n}(0)$, leading to $011010011001011010010110 \ldots$ Dropping its first two digits and partitioning infinite remainder into words of ever increasing even lengths, one writes such infinite sequence as a chain of words $10 ; 1001 ; 10010110 ; 1001011010 \ldots$. where each word can be considered as generating its successor letter by letter in a simple deterministic inflation rule $1 \rightarrow 10,0 \rightarrow 01$. All words in the chain have then a constant density $1 / 2$ and so such must be the infinite chain density.

\section{A.3 More on alternating morphisms scheme}

The (13) version of the O.-K. sequence from Section 3 can also be generated by an equivalent ingenious evolution rule originally proposed in [9] just for the truncated version of the O.-K. sequence written in the $\{1,2\}$ alphabet as $22112122122 \ldots$ (13) of Section 3. We will briefly describe it by returning to $\{0,1\}$ alphabet. One introduces, first, an infinite auxiliary row of labelling indices for sequence elements: $1010101010101010 \ldots$ and declares the following transformation rules:

$$
\mu_{0} ; \mu_{0}(0)=0, \quad \mu_{0}(1)=00
$$

for digits placed in positions 0 and

$$
\mu_{1} ; \mu_{1}(0)=1, \quad \mu_{1}(1)=11
$$

for digits in positions 1 .

One then places digit 1 at the position 1 and claims as it was done in [9] that this is a special seed of the sequence generating word 11 according to (A.2), but still occupying positions 1 and 0 in to be constructed chain. New element 1 in the position 0 then generates a new word: two zeroes occupying positions labelled 1 and 0 . 0 at position 1 then generates 1 to be placed at the first available unoccupied position marked 1 and remaining 0 at position 0 generates 0 to be placed at position 0 . Created in this way word 10 then determines entries to placed at subsequent unoccupied positions forming there word 110, which in turn generates word 11001, etc.

The process can be illustrated by plotting a line of indices with consecutive words placed underneath:

$\begin{array}{llllllllllllll}1 & 0 & 1 & 0 & 1 & 0 & 1 & 0 & \ldots\end{array}$

$11 ; 00 ; 110 ; 1100001 ; \ldots$

The introduction of the seed word was perhaps motivated by the intriguing invariance property of the resulting entire sequence under run length transformation.

Later on, such interest in the sequences coinciding with their run lengths led to sophisticated generalizations for run lengths not necessarily equal to 1 and 2 but operating with any positive integers $r$ and $s$ and alphabet $\{r, s\}$ (see [21] for a review and new results). 
Alternating evolution rules (A.2) and (A.3) are often called alternating morphisms though cannot be reduced to a pure morphism (see Exercise 21 in Chapter 10 of [1]). They can also be used instead of our simple inflation scheme starting from an arbitrary, already cube-free initial word $w_{1}$ by using a simple labelling convention of the subsequent digits of the word $w_{2}$. Namely, if the word $w_{1}$ ends with the block of 1's then the first digit of to be created word $w_{2}$ is labelled with 0 and alternating labels have to be assigned backwards to all digits of $w_{1}$ as well as to all to be created digits at the subsequent steps. Similarly, if the word $w_{1}$ ends with the block of 0 then the first digit of to be created word $w_{2}$ should be labelled with 1 and alternating labels are assigned accordingly to all other positions. In both cases, new position dependent evolution rules are used to evolve $w_{1}$ into $w_{2}, w_{2}$ into $w_{3}$ etc. The key fact in both equivalent methods is the requirement that the created blocks of 1 and 0 must alternate.

\section{A.4 Dekking's rules}

Some years before the paper [9], Dekking [12] had observed that the O.-K. sequence starting from 22 can also be generated by a special transformation rule transforming two-letter words into words of length 2,3 or 4 . In terms of the $\{0,1\}$ alphabet these transformations are:

$$
\delta(00)=10, \quad \delta(1)=1, \quad \delta(10)=110, \quad \delta(11)=1100 .
$$

Dekking also indicated that the rule has to be amended by an additional convention of evolution of words of odd length created in the process. Indeed, starting from 11 as a seed, we obtain $1100|10| 110 \mid$. The last word formed in this process has an odd length and an additional convention says that in all such cases one should disregard last letter of such word and continue expansion according to the preceding it two-letter word.

This will result in $1100|10| 110|1100| 110010|10010110| \ldots$, where again the last created word has odd length.

Returning to the original $\{1,2\}$ alphabet we obtain $2211|21| 221|2211| 221121|22112122| \ldots$, which appears to coincide with the sequence given in Equations (13). Still, one may worry here about formal equivalence proof of both evolution procedures.

\section{A.5 Fibonacci words}

These are words produced by morphisms: $\varphi: \varphi(0)-1, \varphi(1)-10$ and its re-coded variant $\bar{\varphi}: \bar{\varphi}(0)=0, \bar{\varphi}(0)=01$ leading to mirroring each other sequences of iterates: $\varphi^{(n)}(1)$ and $\bar{\varphi}^{(n)}(0): 101101011011010110 \ldots$ and $010010100100101001 \ldots$.

Dropping first two elements of the first of them one can view the rest of it as obtained in the inflation scheme $1 ; 10 ; 101 ; 10110 ; 10110101 ; \ldots$ where subsequent digits of each next word are produced by replacing in the preceding word, digit after digit: 0 by 1 and 1 by 10 . It can also be viewed as a way of extending walks on the graph $11-0-1$ which are started from vertex 1 . Quite similarly, one can modify the second sequence above by dropping its first two digits and start the inflation scheme $0 ; 01 ; 010 ; 01001 ; 01001 ; 01001010 ; \ldots$. First of the sequences never contains squares 00 as sub-words, the mirror sequence does not contain squares 11 . It is well known (see, e.g., [1]) that these sequences have infinite chain densities equal, respectively, $(3-\sqrt{5}) / 2<2 / 3$ and $(\sqrt{5}-1) / 2>1 / 3$. 
Two remarks appear of some significance in this context:

1) "Thinning" of the O.-K. sequence (again without its first two elements) by replacing all 11 squares there by 1 or "compacting" it by replacing all its 00 squares by 0 does not produce Fibonacci words.

2) It could be interesting, however, to apply the above simple inflation schemes to longer initial words without squares of one type and not showing up as any of the words in the Fibonacci chains.

Example: $110101: 1010110110: 1011011010110101: \ldots$, etc.

Question: Do they all have densities coinciding with that for the "denser" Fibonacci word?

\section{References}

[1] Allouche, J.-P., \& Shallit, J. (2003). Automatic Sequences, Cambridge University Press.

[2] Allouche, J.-P., \& Shallit, J. (2003). The ubiquitous Prouhet-Thue-Morse sequence. In: Ding, C., Helleseth, T., \& Niederreiter, H. (eds) Sequences and Their Applications. Proceedings of SETA '98. Discrete Mathematics and Theoretical Computer Science Series. Springer, London. DOI: 10.1007/978-1-4471-0551-0_1. Available online at: http:// ciencias.uis.edu.co/lenguajes/doc/ubiq.pdf.

[3] Bernini, A. (2017). Restricted binary strings and generalized Fibonacci numbers. In: Proceedings of 23th International Workshop on Cellular Automata and Discrete Complex Systems (AUTOMATA), Springer, Vol. 10248, 32-43.

[4] Blanchet-Sadri, F. (2007). Algorithmic Combinatorics on Partial Words. Chapmann \& Hall/CRC.

[5] Bordellès, O., \& Cloitre, B. (2011). Bounds for the Kolakoski sequence. Journal of Integer Sequences, 14, Article 11.2.1.

[6] Capri, A. (1993). Repetitions in the Kolakoski sequence. EATCS Bulletin, 50, 194-196.

[7] Christandl, M., Datta, N., Ekert, A., \& Landhal, A. J. (2004). Perfect state transfer in quantum spin networks. Physical Review Letters, 92(18), Art. No. 187902.

[8] Chvátal, V. (1993). Notes on the Kolakoski Sequence. DIMACS Technical Report 93-84. Available online at: https://users.encs.concordia.ca/ chvatal/93-84. pdf.

[9] Culik, K., \& Karhumäki, J. (1992). Iterative devices generating infinite words. Lecture Notes in Computer Science, 577, 531-544.

[10] Culik, K., Karhumäki, J., \& Lepistö, A. (1992). Alternating iteration of morphisms and the Kolakoski sequence. In: Rozenberg, G., \& Salomaa, A. (eds.), Lindenmayer Systems, Springer, Berlin, 93-106. 
[11] Currie, J., \& Rampersad, N. (2010). Cubefree words with many squares. Discrete Mathematics and Theoretical Computer Science, 12(3), 29-34. Available online at: https://hal.inria.fr/hal-00990439/document.

[12] Dekking, M. (1997). What is the long range order in the Kolakoski sequence? In: Moody, R. V. (ed). The Mathematics of Long-Range Aperiodic Order, Kluwer, Dordrecht, 115-125.

[13] Haeseler, von F. (2008). Automatic Sequences, Berlin, New York: De Gruyter. DOI:10.1515/9783110197969.

[14] Huang, Y B., \& Weakley, W. D. (2010). A note on the complexity of $\mathrm{C} \infty$-words. Theoretical Computer Science, 411(40-42), 3731-3735.

[15] Keane, M. S. (1991). Ergodic theory and subshifts of finite type. In: Bedford, T., Keane, M., \& Series, C. (eds.), Ergodic Theory, Symbolic Dynamics and Hyperbolic Spaces, Oxford University Press, 35-70.

[16] Kolakoski, W. (1965). Self generating runs, Problem 5304. American Mathematical Monthly, 72, 674.

[17] Kuprin, E. J., \& Rowland, E. S. (2008). Bounds on the frequency of 1 in the Kolakoski word. Preprint. Available online at: arXiv:08809.2777v2 [mathCP].

[18] Lucas, A. (2014). Ising formulation of many NP problems. Frontiers in Physics, 2, 5. DOI: 10.3389/fphy.2014.00005.

[19] Oldenburger, R. (1939). Exponent trajectories in symbolic dynamics. Transactions of the American Mathematical Society, 46(3), 453-466.

[20] Paun, G., \& Salomaa, A. (1966). Self-reading sequences. American Mathematical Monthly, 103(2), 166-168. DOI:10.2307/2975113

[21] Sing, B. (2011). More Kolakoski sequences. Integers, 11B, Article No. A14.

[22] Sloane, N. J. A. A000002. The On-Line Encyclopedia of Integer Sequences. Available online at: https://oeis.org/A000002.

[23] Stanley, R. P. (2013). Algebraic Combinatorics. Undergraduate Texts in Mathematics, Springer, New York.

[24] Weisstein, E. W. Kolakoski Sequence. MathWorld-A Wolfram Web Resource. Available online at: https://mathworld.wolfram. com/Kolakoski.Sequence.html.

[25] Wikipedia contributors. (2021). Kolakoski sequence. In: Wikipedia, The Free Encyclopedia. Available online at: https://en.wikipedia.org/w/index.php? title=Kolakoski_sequence\&oldid=1006035333. 